IOSR Journal of Pharmacy

ISSN: 2250-3013, www.iosrphr.org

||| Volume 2 Issue 5 ||| Sep-Oct. 2012 || || PP.26-38

\title{
Liposomes - the potential drug carriers
}

\author{
V.N.L.Sirisha ${ }^{1}$, I.BhavaniHarika, B.Sruthi, M.Namrata, P.Kirankumar, Y.Kiran \\ Kumar Rao, K.Pranavi, S.Sindhura, N.Vamsi Krishna, O.UmaMaheshwaraRao, \\ Anurag Pharmacy College Affiliated to JNTUH, Kodad, Nalgonda-Dt, Andhra Pradesh.
}

\begin{abstract}
Liposomes have been widely investigated since 1970 as drug carriers for improving the delivery of therapeutic agents to specific sites in the body. As a result, numerous improvements have been made, thus making this technology potentially useful for the treatment of certain diseases in the clinics. The success of liposomes as drug carriers has been reflected in a number of liposome-based formulations, which are commercially available or are currently undergoing clinical trials. This review is focused on the various aspects of liposomes starting from the evolution and the current research including the detailed summary such as classification, methods of preparation, characterization and the potential use of liposomes in the treatment of various diseases and the various sites to which these are targeted.Liposomes have covered predominantly medical, albeit some non-medical areas like bioreactor, catalysts, cosmeticsand ecology. However, their predominance in drug delivery and targeting has enabled them to be used astherapeutics tool in fields like tumor targeting, gene and antisense therapy etc.
\end{abstract}

Keywords—Characterization, Constraints, Industrial production, Liposomes, Phospholipids

\section{INTRODUCTION ${ }^{[2]}$}

The rising number of complications associated with drugs from varied chemical and biological background not only made scientists worldwide to search for newer molecules but also to discover the new ways and means for the proper delivery of molecules. With the help of new delivery systems known as novel drug delivery systems [NDDS] both old and new molecules can be delivered to the site in demand in a defined manner. With this targeted delivery, the molecules can be made to produce the desired effect without disturbing the delicate bio-environment. The investment on drug delivery research in contrast to search for the basic therapeutic molecules of synthetic origin may well prove to be less taxing in terms of money, labor and time. This led to the development of an array of approaches based on varied physicochemical and biological tools and techniques which can be used to deliver the drug to the desired targeted site with reduced or no toxicity. Among many available colloidal drug delivery systems, a class based on phospholipids has fetched much more attention than the other systems because of its meritorious

Features. These vesicular systems have displayed their potential to a great extent in delivering thevarious drugs to the target site i.e., Liposomes. These have emerged as most practically usefulcarriers for invivo drug delivery as majority of reports has concentrated on the use of phospholipid vesicles or liposomes as potential drug carrier systems. ${ }^{[2]}$

\subsection{Evolution of liposomes ${ }^{[2]}$}

The history of liposomes goes back to mid-1960's and the credit of their birth goes to Bangham and his coworkers, who discovered that phospholipids in presence of suitable solvents form bi-layered membranes which finally curl-on to form uni-lamellar or multi-lamellar vesicles. The history of liposomes can be divided into three periods:

\subsubsection{Genesis [1968-75]}

The physiochemical characterization of liposomes had been carried out in this period. Moreover, thin lipid film hydration method had been developed to prepare multi-lamellar vesicles [MLVs].

\subsubsection{Middle Age [1975 - 85]}

Liposome's utility was improved following basic research that increased the understanding of their stability and interaction characteristic within the system. This period also dealt with thediscovery of various alternative methods for the preparation of liposomes. Also, due to the availability of vast knowledge about the physio-chemical properties of liposomes, their behavior within the body, their interaction with the cells, attempts had been made to improve their performance as drug carrier systems. 


\subsubsection{Modern Era [1985 onwards]}

Today, liposomes are used successfully in various scientific disciplines, including mathematics and theoretical physics [topology of two-dimensional surfaces floating in a three dimensionalcontinuum], biophysics [properties of cell membranes and channels], chemistry [catalysis,energy conversion, photosynthesis], colloid science [stability, thermodynamic of finite systems], biochemistry [function of membrane proteins] and biology [excretion, cell function, trafficking and signaling, gene delivery and function].Moreover, renaissance in the liposome research is promising many more products to come in the near future. ${ }^{[2]}$

\subsection{Definition ${ }^{[1]}$}

"Liposomes are simple microscopic vesicles in which an aqueous volume is entirely enclosed bya membrane composed of lipid molecule." Various amphipathic molecules have been used to form liposome. The drug molecules can either be encapsulated in aqueous space or intercalated into the lipid bilayer.It is a spherical vesicle with a membrane composed of a phospholipid bilayer used to deliver drug or genetic material into a cell.

These can be composed of naturally-derived phospholipids with mixed lipid chain like eggphosphatidylethonalimine or of pure components like DOPE [dioleolylphosphatidylethanolamine]. ${ }^{[1]}$

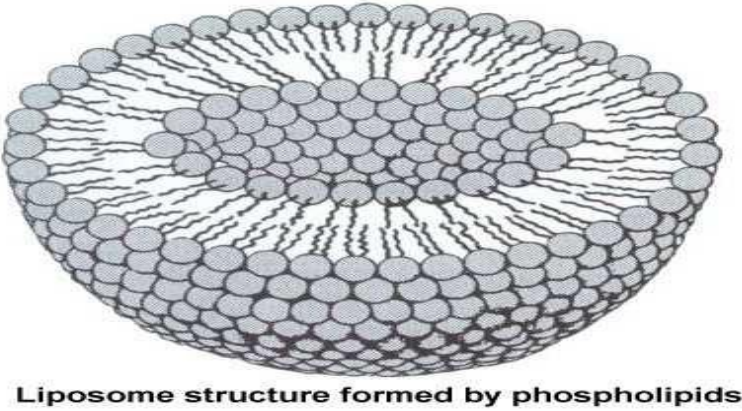

Fig.1. Liposomal Structure

\subsubsection{Advantages ${ }^{[1]}$}

- Provides selective passive targeting to tumor tissue[liposomal doxorubicin]

- Liposomes have increased efficacy and therapeuticindex of drug [Actinomycin-D].

- Liposomes have increased stability via encapsulation.

- Liposomes are biocompatible, completely biodegradable, non-toxic, flexible andnon-immunogenic for systemic and non-systemicadministrations.

- Liposomes have reduction in toxicity of the encapsulated agent [Amphotericin B, Taxol].

- Liposomes help to reduce exposure of sensitivetissues to toxic drugs.

- Site avoidance effect.

- Flexibility to couple with site-specific ligands toachieve active targeting.

\subsubsection{Disadvantages ${ }^{[1]}$}

- Production cost is high.

- Leakage and fusion of encapsulated drug /molecules.

- Sometimes phospholipid undergoes oxidation andhydrolysis like reaction.

- Short half-life.

- Low solubility

- Inadequate stability due to uptake by Reticuloendothelial system.

\section{3. Structural Components of liposomes ${ }^{[2]}$ \\ 1.3.1. Membrane forming components \\ 1.3.1.1. Phospholipids: Bilayer formers}

Phospholipids that are the major components of the biological membranes are the building blocks of the liposomes. The phospholipids have tubular shape owning to the presence of two acyl chains attached to a polar head and on hydration, results into a bilayered membrane. Two types of phospholipids are there i.e. phosphodiglycerides and sphingolipids along with their corresponding hydrolysis products.

\section{Classification of phospholipids}

- Neutral phospholipids e.g. Sphingomyelin, Phosphatidylethanolamine and Phosphatidylcholine.

- Negatively charged phospholipids e.g. Dipalmitoylphosphatidylcholine, Dipalmitoylphosphatidyl acid [DDPA], Distearoylphosphatidyl choline [DSPC], Dioleoylphosphatidyl choline [DOPC] etc.

- Positively charged phospholipids e.g. 1, 2-dihexadecyl-N, N-dimethyl-N-trimethyl amine methyl ethanol amineetc. 


\subsubsection{Membrane Additives [Sterols]}

Cholesterol is the most commonly used sterol, which is included in the liposomal membranes. It has been called as the 'motar' of bilayers because by virtue of its molecular shape and solubility properties, it fills in empty spaces among the phospholipid molecules, anchoring them more strongly into the structure. Cholesterol is an amphipathic molecule and inserts itself into the membrane with its hydroxyl groups oriented towards the aqueous phase and aliphatic chain aligned parallel to acyl chains of the phospholipid molecules. In other words, cholesterol increases the transition temperature of the system by making the membrane more ordered. Cholesterol reduces this type of interaction to a great extent and provides both physical and biological stability.

\subsubsection{Charge inducers and steric stabilizers}

Stearylamine, dicetylphosphate, solulan C-24 and diacylglycerol are commonly used to impart eithera negative or a positive surface charge. Since it is a well-known fact that negatively charged and positively charged liposomes are more rapidly uptaken by the reticulo-endothelial system as compared to neutral liposomes, charge inducers are used to overcome this problem. Also they proved to be useful in reducing aggregation as neutral liposomes show higher tendency to undergo aggregation.

\subsubsection{Other substances:}

In case, the drug is very prone to oxidation, antioxidants e.g. tocopherol, butylatedhydroxy toluene and stabilizers are used. The use of preservatives is very common to increase the shelf-life of liposomal formulations. ${ }^{[2]}$

\subsubsection{Mechanism of liposome formation: ${ }^{[1]}$}

Phospholipids are amphipathic having affinity for bothaqueous and polar moieties molecules as they have ahydrophobic tail and a hydrophilic or polar head. Thehydrophobic tail is composed of two fatty acid chaincontaining 10-24 carbon atom and 0-6 double bonds ineach chain. The macroscopic structures most oftenformed include lamellar, hexagonal or cubic phasesdispersed as colloidal nanoconstructs [artificialmembranes] referred to as liposome's, hexasomes orcubosomes. The most common natural polar phospholipids arephosphatidylcholine. These are amphipathic moleculesin which a glycerol bridge links to a pair of hydrophobic acyl hydrocarbon chains with a hydrophilic polar head group, phosphocholine. The amphipathic nature of phospholipids and their analogues render them the ability to form closed concentric bilayers in presence of water. Liposomes are formed when thin lipid films or lipid cakes are hydrated and stacks of lipid crystalline bilayers become fluid and swell. The hydrated lipid sheets detach during agitation and selfclose to form large, multi-lamellar vesicles prevent interaction of water with the hydrocarbon core of the bilayer at the edges.

\subsection{Formulation factors affecting the degreeof drug entrapment: ${ }^{[3]}$}

The extents of drug entrapment and retention as well as factors influencing them areimportant considerations in the design ofliposome-mediated drug delivery systems.Drugs may be entrapped in the aqueous and/or lipid phase of the liposome.

\subsubsection{Aqueous entrapment}

This relates to the aqueous volume in the liposome. The larger the aqueous volume the greater the amounts of polar drugs that canbe encapsulated. Multiple compartmentliposomes encapsulate higher percentages of aqueous soluble drugs than single compartment vesicles, because of the larger volume of encapsulated aqueous space in the former. Formulations that promote formation of MLVs are thus associated with higher aqueous entrapment. Osmoticswelling and/or incorporation of charged lipids, e.g., phosphatidylserineinto bilayers are measures forincreasing the aqueous volume in liposomes. The latter is due to charge repulsionseparating adjacent bilayers, resulting inincreases in trapped aqueous volume.Aqueous solubility of the drug is another factor, hence, the extent of drug entrapmentin liposomes [MLVs] can vary markedly as seen in the following examples: $2.2-8.4 \%$ forpenicillin, $2.3-11.6 \%$ for actinomycin $\mathrm{D}, 18 \%$ for methothrexate and up to $60 \%$ for bleomycin. Leakage of entrapped solute is another formulation problem. Cholesterol modifies the fluidity of lipid membranes, thereby influencing the degree of retention of drugs by vesicles as well as stabilising thesystem against enzymatic degradation. Largemolecules [e.g., peptides and proteins] are better retained than smaller molecules, which can diffuse slowly through the lipid layers. ${ }^{[3]}$

\subsubsection{Lipid entrapment}

Lipid soluble drugs are entrapped in the lipid layers of liposome. Here, the entrapment efficiency can be as high as $100 \%$, irrespective of liposomal type and composition. An example of a drug that is hydrophobic innature is camptothecin.The retention ofsuch hydrophobic drugs is also high when the liposomes are placed in aqueous biological environment because of their high lipid-water partition coefficients. ${ }^{[3]}$

\subsection{Preparation methods ${ }^{[28]}$}

1. Active Loading Technique

2. Passive Loading Techniques are as follows: ${ }^{[28]}$ 
Table.1. List of various methods of preparation of liposomes.

\begin{tabular}{|c|c|c|}
\hline $\begin{array}{l}\text { MechanicalDispersion } \\
\text { methods }\end{array}$ & Solvent Dispersion methods & Detergent Removal methods \\
\hline $\begin{array}{ll}\text { - Lipid film hydration- } \\
\text { Hand shaking, Non } \\
\text { Hand shaking, Freeze } \\
\text { Drying } \\
\text { - Micro emulsification } \\
\text { - Sonication } \\
\text { - French Pressure Cell } \\
\text { - Membrane extrusion } \\
\text { - Dried reconstituted } \\
\text { - } \text { vesicles } \\
\text { Freeze-thawed } \\
\text { liposomes }\end{array}$ & $\begin{array}{ll}\text { - } & \text { Ethanol injection } \\
\text { - } & \text { Ether Injection } \\
\text { - } & \text { Double Emulsion } \\
& \text { Vesicles } \\
\text { - } & \text { Reverse Phase } \\
\text { evaporation } & \\
\text { vesicles } \\
\text { - Stableplurilamellar } \\
\text { vesicles }\end{array}$ & $\begin{array}{l}\text { Detergent Removal from } \\
\text { mixed micelles by } \\
\text { - } \\
\text { - } \\
\text { - } \\
\text { Dialysis } \\
\quad \text { colution } \\
\text { chromatography }\end{array}$ \\
\hline
\end{tabular}

Some of the methods of preparation are described as follows: ${ }^{[4]}$

\subsubsection{Lipid Hydration Method}

This is the most widely used method for the preparation of MLV. The method involves drying a solution of lipids so that a thin film is formed at the bottom of round bottom flask and then hydrating the film by adding aqueous buffer and vortexing the dispersion for some time. The hydration step is done at a temperature above the gel-liquid crystalline transition temperature of the lipid or above the Tc of the highest melting component in the lipid mixture. The compounds to be encapsulated are added either to aqueous buffer or to organic solvent containing lipids depending upon their solubilities.MLV are simple to prepare by this method and a variety of substances can be encapsulated in these liposomes. The drawbacks of the method are low internal volume, low encapsulation efficiency and the size distribution is heterogeneous.

\subsubsection{Solvent Dispersion Method}

A method for the preparation of MLVs of homogeneous size distribution wasproposed by Kim et al. The process involved dispersing in aqueous solution thesmall spherules of volatile hydrophobic solvent in which lipids had been dissolved.MLVs were formed when controlled evaporation of organic solvent occurred in a waterbath.

\subsubsection{Sonication Method}

Here MLVs are sonicated either with a bath type sonicator or a probe sonicator underan inert atmosphere. The main drawbacks of this method are very low internalvolume/encapsulation efficiency, possibly degradation of phospholipids and compoundsto be encapsulated, exclusion of large molecules, metal contamination from probe tip andpresence of MLV alongwith SUV.

Recently, Oezden and Hasirci[1991] prepared apolymer coated liposomes by this method.

\subsubsection{French Pressure Cell Method}

The method involves the extrusion of MLV at 20,000 psi at $4^{\circ} \mathrm{C}$ through a smallorifice. The method has several advantages over sonication method. The method issimple rapid, reproducible and involves gentle handling of unstable materials [Hamiltonand Guo, 1984]. The resulting liposomes are somewhat larger than sonicated SUVs. Thedrawbacks of the method are that the temperature is difficult to achieve and the workingvolumes are relatively small [about $50 \mathrm{~mL}$ maximum].

\subsubsection{Solvent Injection Methods \\ 1.5.5.1. Ether Infusion Method}

A solution of lipids dissolved in diethyl ether or ether/methanol mixture is slowlyinjected to an aqueous solution of the material to be encapsulated at $55-65^{\circ} \mathrm{C}$ or underreduced pressure. The subsequent removal of ether under vacuum leads to the formationof liposomes. The main drawbacks of the method are that the population is heterogeneous [70-190 nm] and the exposure of compounds to be encapsulated to organic solvents orhigh temperature [Dcamcr and Bangham, 1976; Schieren et al., 1978].

\subsubsection{Ethanol Injection Method}

A lipid solution of ethanol is rapidly injected to a vast excess of buffer. The MLVsare immediately formed. The drawbacks of the method are that the population isheterogeneous [30-110 nm], liposomes are very dilute, it is difficult to remove all ethanolbecause it forms azeotrope with water and the possibility of various 
biologically activemacromolecules to inactivation in the presence of even low amounts of ethanol [BatzriandKorn, 1973].

\subsubsection{Reverse Phase Evaporation Method}

First water in oil emulsion is formed by brief sonication of a two phase system containing phospholipids in organic solvent [di-ethylether or isopropylether or mixture of isopropyl ether and chloroform] and aqueous buffer. The organic solvents are removed under reduced pressure, resulting in the formation of a viscous gel. The liposomes are formed when residual solvent is removed by continued rotary evaporation under reduced pressure. With this method high encapsulation efficiency up to $65 \%$ can be obtained in a medium of low ionic strength for example $0.01 \mathrm{M} \mathrm{NaCl}$. The method has been used to encapsulate small, large and macromolecules. The main disadvantage of the method is the exposure of the materials to be encapsulated to organic solvents and to brief periods of sonication. These conditions may possibly result in the denaturation of some proteins or breakage of DNA strands [Szoka and

Papahadjopoulos, 1978]. We get a heterogeneous sized dispersion of vesicles by this method. Modified Reverse Phase Evaporation Method was presented by Handa et al. [1987] and the main advantage of the method is that the liposomes had high encapsulation efficiency[about 80\%].

The Reverse Phase Evaporation Method of Szoka and Papahadjopoulos[1978]have also been modified to entrap plasmids without damaging DNA strands [Haga and Yogi, 1989].

\subsubsection{Detergent Removal Methods}

The detergents at their critical micelles concentrations have been used to solubilizelipids. As the detergent is removed the micelles become progressively richer inphospholipid and finally combine to form LUVs. The detergents were removed bydialysis [Kagawa and Rocker, 1971; Milsmann et al., 1978; Alpes et al., 1986]. The advantagesof detergent dialysis method are excellent reproducibility and production ofliposome populations which are homogenous in size. The main drawback of the methodis the retention of traces of detergent[s] within the liposomes. A commercial device calledLIPOPREP [Diachema AG, Switzerland] which is a version of dialysis system isavailable for the removal of detergents. Other techniques have been used for the removalof detergents: [a] by using Gel Chromatography involving a column of Sephadex G-25[Enoch and Suitt matter, 1979], [b] by adsorption or binding of Triton X-100 [adetergent] to Bio-Beads SM-2 [Gerristen et al., 1978]. [c]by binding of octylglucoside[a detergent] to Amberlite XAD-2 beads [Philippot et al., 1985]. ${ }^{[4]}$

\subsection{Industrial Production of Liposomes ${ }^{[4]}$}

Of the several preparation methods described in the literature, only a few havepotential for large scale manufacture of liposomes. The main issues faced to formulatorand production supervisor are presence of organic solvent residues, physical andchemical stability, pyrogen control, sterility, size and size distribution and batch to batchreproducibility.Liposomes for parenteral use should be sterile and pyrogen free. For animal experiments, adequate sterility can be achieved by the passage of liposomes through up toapproximately $400 \mathrm{~nm}$ pore size Millipore filters. For human use, precautions for sterilitymust be taken during the entire preparation process: that is, the raw materials must besterile and pyrogen free, preparation in sterile system: working areas equipped withlaminar flow and use of sterile containers [Freise, 1984].Some issues related to phospholipids need attention. The liposomes based on crudeegg yolk phospholipids are not very stable. The cost of purified lipids is very high.Recently, liposomes have been prepared using synthetic [Yamauchi et a1., 1994] andpolymerizable lipids [Fiona et al., 1987]. The liposomes prepared from polymerizablephospholipids are exposed to UV light. The polymerization process takes place in thebilayer[s]. Such liposome preparations usually have better storage stability. It should benoted that such materials usually are phospholipid analogues and their metabolic fateshave yet to be established. ${ }^{[4]}$

The various methods meant for the industrial production of liposomes are:

\subsubsection{Detergent Dialysis}

A pilot plant under the trade name of LIPOPREPR II-CIS is available fromDiachema, AG, and Switzerland. The production capacity at higher lipid concentration $[80 \mathrm{mg} / \mathrm{ml}]$ is $30 \mathrm{ml}$ liposomes/minute. But when lipid concentration is $10-20 \mathrm{mg} / \mathrm{ml} 100 \mathrm{mg} / \mathrm{ml}$ then up to many litres of liposomes can be produced. In USA, LIPOPREPR ismarketed by Dianorm-Geraete[Maierhofer, 1985].

\subsubsection{Microflluidization}

A method based on micro0uidization/microemulsiftcation/homogenization wasdeveloped for the preparation of liposomes. MICROFLUIDIZERR is available fromMicro0udics Corporation, Massachusetts, USA. A plot plant based on this technology canproduce about 20 gallon/minute of liposomes in 50-200 nm size range. The encapsulationefficiency up to $75 \%$ could be obtained

1.6.3. Aqueous dispersions of liposomes often have tendency to aggregate or fuse and mayhe susceptible to hydrolysis and or oxidation. Two solutions have been proposed: 


\subsubsection{Pro-liposomes}

In pro-liposomes, lipid and drug are coated onto a soluble carrier to form free-flowinggranular material which on hydration forms an isotonic liposomal suspension. Theproliposome approach may provide an opportunity for cost-effective large scalemanufacture of liposomes containing particularly lipophilic drugs [Payne et al., 1986].

\subsubsection{Lyophilization}

Freeze-drying [lyophilization] involves the removal of water from products in thefrozen state at extremely low pressures. The process is generally used to dry products thatare thermolabile and would be destroyed by heat-drying. The technique has a greatpotential as a method to solve long term stability problems with respect to liposomalstability. It is exposed that leakage of entrapped materials may take place during theprocess of freeze- drying and on reconstitution. Recently, it was shown that liposomeswhen freeze-dried in the presence of adequate amounts of trehalose[a carbohydratecommonly found at high concentrations in organism] retained as much as $100 \%$ of theiroriginal contents. It shows that trehalose is an excellent cryoprotectant[freeze-protectant]for liposomes [Crowe et al., 1987]. Freeze-driers ranges in size from small laboratory models to large industrial units are available from Pharmaceutical Equipment Suppliers.Recently Schrier et al. [1994] have studied the in vitro performance of formulationsprepared from lyophilized liposomes. ${ }^{[4]}$

\subsection{Classificationof Liposomes ${ }^{[28]}$ \\ 1.7.1. Based on structural parameters}

- Multi-lamellar large vesicles- $>0.5 \mu \mathrm{m}$-MLV

- Oligo-lamellar vesicles- 0.1-1 $\mu \mathrm{m}-\mathrm{OLV}$

- Uni-lamellar vesicles [all size range]-UV

- Small Uni-lamellar vesicles- 20-100nm-SUV

- Medium sized Uni-lamellar vesicles-MUV

- Large Uni-lamellar vesicles- >100-LUV

- Giant Uni-lamellar vesicles- $>1 \mu \mathrm{m}-\mathrm{GUV}$

- Multi-vesicular vesicles- $1 \mu \mathrm{m}-\mathrm{MV}$

\subsubsection{Based on method of liposome preparation ${ }^{[28]}$}

- Single or oligo-lamellar vesicles made by reverse phase evaporation method-REV

- Multi lamellar vesicles made by reverse phase evaporation method-MLV-REV

- Stable pluri-lamellar vesicles-SPLV

- Frozen and thawed MLV-FATMLV

- Vesicles prepared by extrusion technique-VET

- Dehydration-rehydration method-DRV

\subsubsection{Based on composition and application ${ }^{[28]}$}

- Neutral or negatively charged phospholipids and cholesterol - Conventional liposomes [CL]

- Reconstituted sendai virus envelopes - Fusogenic liposomes [RSVE]

- Phospholipid such as PE or DOPE with either CHEMS or OA- pH sensitive liposomes

- Cationic lipids with DOPE - Cationic liposomes

- Neutral high Tc ${ }^{0}$, Cholesterol and 5-10\% of PEG-DSPE or GMI - Long circulatory liposomes [LCL ${ }^{[16]}$

- $\mathrm{CL}$ or LCL with attached monoclonal antibody or recognition sequence-Immunoliposomes ${ }^{[28]}$

- Phospholipids with alcohol [ethanol and isopropyl alcohol] - Ethosomes ${ }^{[11]}$

- Phospholipids with surfactant mixture - Transferosomes ${ }^{[15]}$

- Phospholipids coated with chitosan - Chitosomes $^{[7]}$

1.8. Characterization of Liposomes ${ }^{[2],[28]}$ 
Table.2: characterization of liposomes.

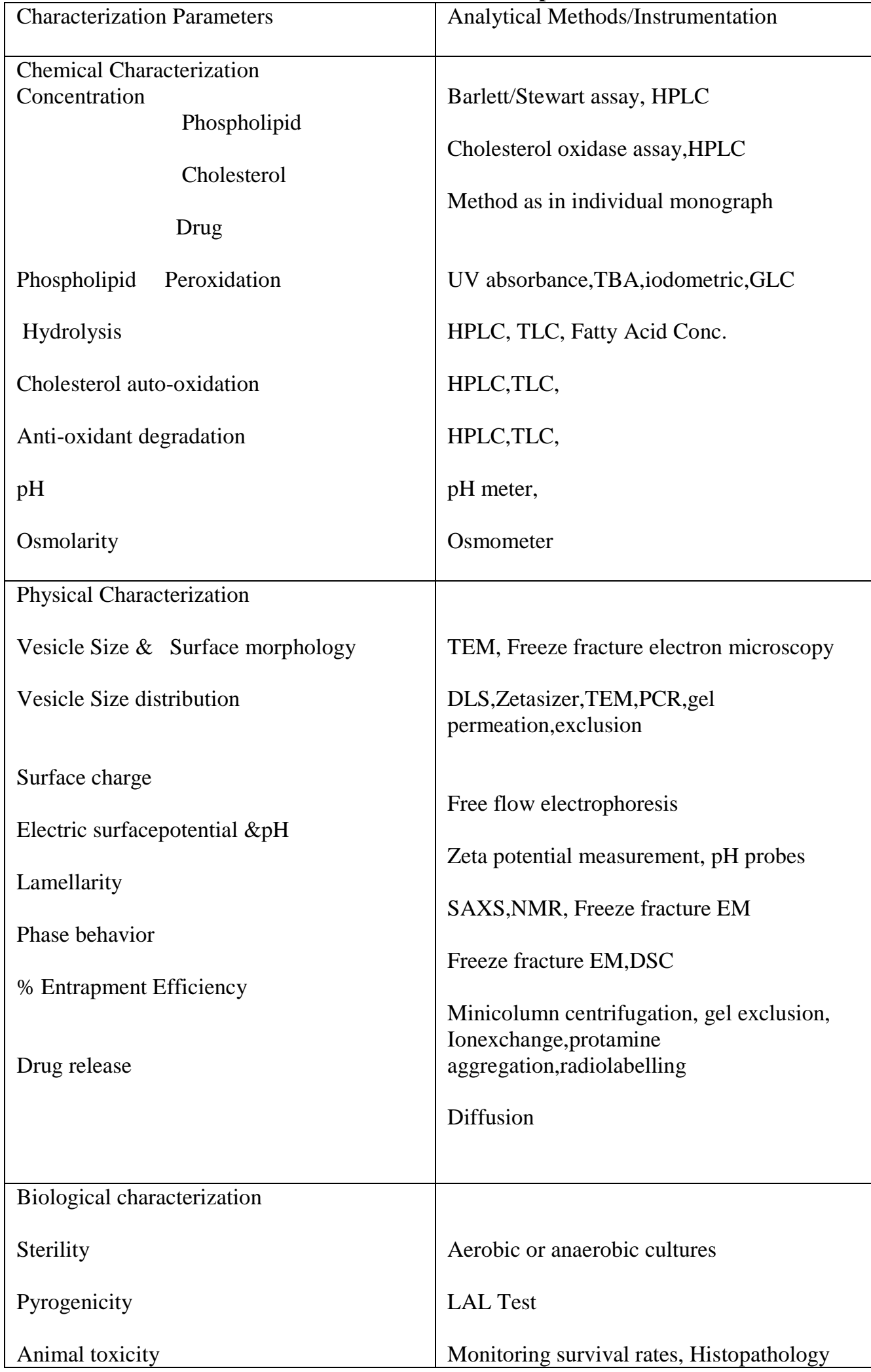

The most important parameters of liposome characterization include visual appearance,turbidity,size distribution, lamellarity, concentration, composition, presence of degradation products, and stability. ${ }^{[1]}$ 


\subsubsection{VisualAppearance ${ }^{[1]}$}

Liposome suspension can range from translucent tomilky, depending on the composition and particlesize. If the turbidity has a bluish shade this means thatparticles in the sample are homogeneous; a flat, graycolor indicates that presence of a non-liposomaldispersion and is most likely a disperse inversehexagonal phase or dispersed micro crystallites. Anoptical microscope [Phase contrast] can detectliposome $>0.3 \mu \mathrm{m}$ and contamination with largerparticles.

\subsubsection{Determination of Liposomal Size Distribution ${ }^{[1]}$}

Size distribution is normally measured by dynamiclight scattering. This method is reliable for liposomeswith relatively homogeneous size distribution. Asimple but powerful method is gel exclusion chromatography, in which a truly hydrodynamic radius can be detected. Sephacryl-S100 can separate liposome in size range of 30-300nm. Sepharose $-4 \mathrm{~B}$ and $-2 \mathrm{~B}$ columns can separate SUV from micelles.

\subsubsection{Determination of Lamellarity ${ }^{[1]}$}

The lamellarity of liposomes is measured by electron microscopy or by spectroscopic techniques. Most frequently the nuclear magnetic resonance spectrum of liposome is recorded with and without the addition of a paramagnetic agent that shifts or bleaches the signal of the observed nuclei on the outer surface of liposome. Encapsulation efficiency is measured by encapsulating a hydrophilic marker.

\subsubsection{Liposome Stability ${ }^{[1]}$}

Liposome stability is a complex issue, and consists ofphysical, chemical, and biologicalstability. In thepharmaceutical industry and in drug delivery, shelf lifestability is also important. Physical stabilityindicates mostly the constancy of the size and the ratioof lipid to active agent. The cationic liposomes can bestable at $4{ }^{\circ} \mathrm{C}$ for a long period of time, if properlysterilized.

\subsubsection{Entrapped Volume ${ }^{[1]}$}

The entrapped volume of a population of liposome [in $\mu \mathrm{L} / \mathrm{mg}$ phospholipid] can often be deduced frommeasurements of the total quantity of solute entrappedinside liposome assuring that the concentration ofsolute in the aqueous medium inside liposomes is thesame after separation from unentrapped material. Forexample, in two phase method of preparation, watercan be lost from the internal compartment during thedrying down step to remove organic solvent.

\subsubsection{Surface Charge ${ }^{[1]}$}

Liposomes are usually prepared using charge impartingconstituting lipids and hence it is imparting to studythe charge on the vesicle surface. In general twomethod are used to assess the charge, namely freeflowelectrophoresis and zeta potential measurement.From the mobility of the liposomal dispersion in asuitable buffer, the surface charge on the vesicles.

\subsection{Pharmacokinetics ${ }^{[3]}$}

Most small molecular chemotherapeuticagents have a large volume of distribution onintravenous [IV] administration of liposomes. The result of this wide distribution is often anarrow therapeutic index due to a high levelof toxicity on healthy tissues. Throughencapsulation of drugs in liposomes, thevolume of distribution is significantlyreduced and the concentration of drug at thedesired site of action increased. For instance,liposomal drug delivery led to an increase inthe amount of drug that can be effectivelydelivered to tumor sites in anticancertherapy. Liposomes are predominantlyremoved from circulation by phagocyte cellsof the reticuloendothelial system [RES], thusaccumulating to a large extent in organs likeliver and spleen. This biodistribution patterncan be used for passive targeting of diagnosticsto these organs. The RES should, therefore,be saturated with empty vesicles when othersites are the drug targets. Information onbio-distribution is, therefore, important for

Drug targeting by liposomes.Liposomes given intravenously usuallyinteract with at least two distinct groups ofplasma proteins. These are the plasma highdensity lipoproteins and the so-calledopsonins, which bind to the surface of vesiclesand mediate their endocytosis by themononuclear phagocyte system [macrophages].The rate of liposome clearance from bloodcirculation will, therefore, depend on theability of opsonins to bind to the liposome

Surface. The rate can be manipulated throughappropriate selection of liposome characteristics. For instance, "fluid" vesicles areremoved more rapidly from blood circulationthan "rigid" ones. Clearance from the bloodstream is also influenced by vesicle size andsurface charges. The longest half-life isobtained when liposomes are relatively small

[diameter $<0.05 \mu \mathrm{m}$ ] and carry no net surfacecharge. The pharmacokinetic behaviour ofliposomes depends on the route of injectionsuch as intraperitoneal, subcutaneous orintramuscular route. Lasic and Papahadjopouloshave shown that coating theliposome surface with polyethylene glycol andother hydrophobic part of phospholipids substantially prolongs the half-life ofliposomes in the blood. 


\section{STORAGE OF LIPOSOMES: FREEZE-DRYING ${ }^{[3]}$}

Liposome dispersions are potentially prone tohydrolytic degradation and leakage. Hence, itis desirable to freeze-dry the suspension to apowder and store in this dried form. Thepowder can be reconstituted to an aqueoussuspension immediately before use. By doingso, SUVs may be converted to MLVs dispersionupon rehydration. Addition of a carbohydrate[trehalose] during freeze-drying preventsfusion and leakage of the vesicles.

\subsection{Physical stability ${ }^{[1]}$}

\section{STABILITY}

There is no established protocol foreither accelerated or long-term stability studies for theliposomal formulation. Classical models from colloidalscience can be used to describe liposome stability.Colloidal systems are stabilized electrostatically,sterically or electrosterically. In addition the self-assemblingcolloids can undergoes fusion or phasechange after aggregation. Liposome exhibit bothphysical and chemical stability characteristics.Generally, the physical characteristic describes thepreservation of liposome structure and the chemicalcharacteristic refers to molecular structure of liposomalcomponents. [hydrolysis and oxidation ofphospholipid] Physically stable formulations preserveboth liposome size distribution and the amount ofmaterial encapsulated. The stability problemovercomes by using appropriate techniques likefreezing, lyophilization and osmification. It is alsoprevented by using fresh solvents and freshly purifiedlipid, using inert nitrogen gas, avoid high temperature

and include anti-oxidants.

\subsection{Temperature studies}

High-temperaturetesting [greater than $25^{\circ} \mathrm{C}$ ] is almost universally usedfor heterogeneous products. For liposomes,elevated temperatures may dramaticallyalter the nature of the interfacial film,especially if the phasetransition temperatureis reached. If one expects the productto be exposed to a temperature of $45^{\circ} \mathrm{Cfor}$ extended periods [or even for shortdurations], studies at $45-50^{\circ} \mathrm{C}$ are quitejustified. Studying a liposomal product atsuch temperatures determines how theproduct will hold up and whether anydamage is reversible when the product isbrought back to room temperature. Iftemperatures higher than the system willever encounter are used even in shorttermheat-cool cycling one risks irreversiblydamaging the bilayers such thatthe membrane cannot heal when broughtback to room temperature. If a liposomal dispersion is partiallyfrozen and then thawed, ice crystals nucleateand grow at the expense of water.Liposomes then may press together againstthe ice crystals under great pressure. Ifcrystals grow to sizes greater than the voidspaces, instability is more likely. That phenomenonis well noted with a slower rateof cooling, causing formation of larger icecrystals, which leads to greater instability.Certain polymers are known to retard icecrystal growth. ${ }^{[9]}$

\subsection{Plasma Stability ${ }^{[1]}$}

Although liposomes resemble biomembranes, they stillare foreign objects for the host.Therefore, liposomesare recognized by the mononuclear phagocytic system[MPS] after interaction with plasma proteins. As aresult, liposomes are cleared from the blood stream.These stability problems solve by using syntheticphospholipids, gangliosides, polymerization, coating liposomes with chitin derivatives, freeze drying,microencapsulation and particle coated with amphipathic polyethylene glycol.

\section{VARIOUS TECHNOLOGIES OF LIPOSOMES FOR SPECIALIZED DRUG DELIVERY ${ }^{[16]}$}

\section{1 liposome-Based Technology}

- Conventional liposomes

- Stealth liposomes

- Targeted liposomes

- Other types of liposomes such as virosomes and gene based liposomes 


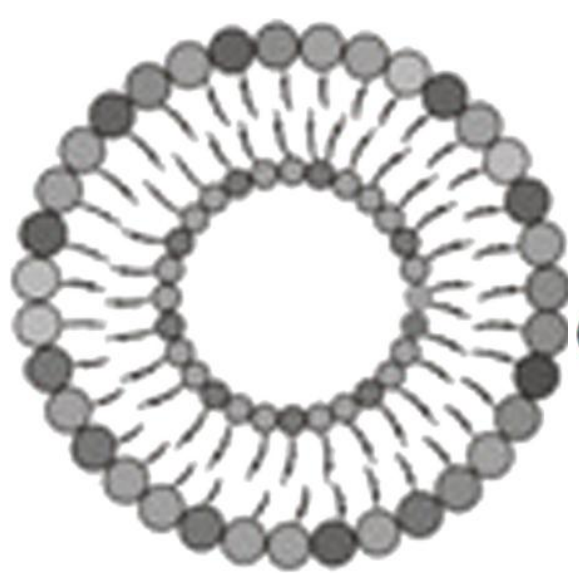

Fig. 2.Conventional Liposome $e^{[16]}$

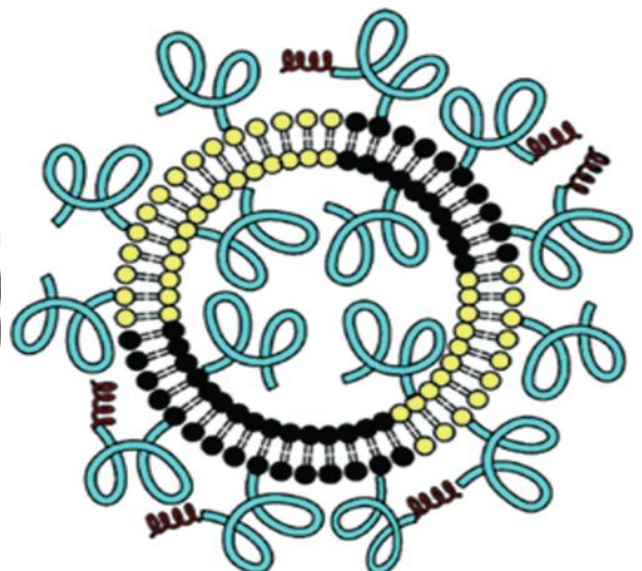

Fig. 3.PEGylated Liposome ${ }^{[16]}$

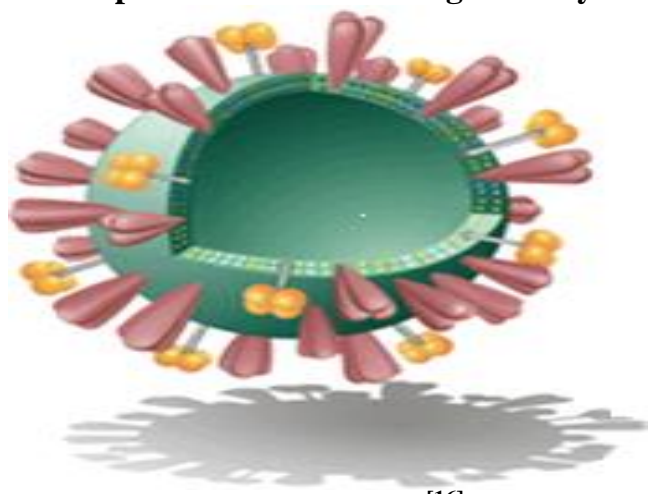

Fig: 4 Virosome ${ }^{[16}$

\section{VARIOUS LIPOSOMAL DRUG DELIVERIES}

\subsection{Temporary Depot Polymeric-Based Systemsfor Liposomal Coupling}

- Injectable Polymeric Scaffolds.

- Prefabricated Polymeric Scaffolds.

5.2. Natural Product-Based Liposomal Drug Delivery Systems

- Collagen-Based Liposomal Drug Delivery Systems

- Gelatin-Based Liposomal Drug Delivery Systems.

- Chitosan-Based Liposomal Drug Delivery Systems.

- Fibrin-Based Liposomal Drug Delivery Systems.

- Alginate-Based Liposomal Drug Delivery Systems.

- Dextran-Based Liposomal Drug Delivery Systems.

5.3. Liposomal Drug Delivery Systems Based on Synthetic Polymers

- Carbopol-Based Liposomal Drug Delivery Systems

- Polyvinyl Alcohol-Based Liposomal Drug Delivery Systems

5.4. Techniques for Embedding Drug-Loaded Liposomes within Depot Polymeric-Based systems:

5.5. ModulatingDrug Release fromLiposomes within Polymeric Depot Systems

$$
\text { VI. APPLICATIONS: }{ }^{[1],[16]}
$$

\subsection{Liposome as drug/protein delivery vehicles}

- Controlled and sustained drug release

- Enhanced drug solubilization

- Altered pharmacokinetics andbio-distribution

- Enzyme replacement therapy andbio-distribution

- Enzyme replacement therapy and lysosomalstorage disorders

6.2. Liposome in anti-microbial,antifungal and antiviraltherapy

- Liposomal drugs

- Liposomal biological response modifiers 


\subsection{Liposome in tumor therapy}

- Carrier of small cytotoxic molecules

- Vehicle for macromolecules as cytokines orgenes

6.3. Liposome in gene delivery

- Gene and antisense therapy

- Genetic [DNA] vaccination

6.4. Liposome in immunology

- Immunoadjuvant

- Immunomodulator

- Immunodiagnosis

6.5. Liposome as artificial blood surrogates

6.6. Liposome as radiopharmaceutical and radiodiagnostic carriers

6.7. Liposome in cosmetics and dermatology

6.8. Liposome in enzyme immobilization andbioreactor technology

\section{MISCELLANEOUS APPLICATIONS}

- PEGylated Liposomal doxorubicin in the treatment of ovarian cancer. ${ }^{[6]}$

- Liposomal daunorubicin in the treatment of leukaemia. ${ }^{[5]}$

- Liposomal neomycin and penicillin are widely used in antimicrobial therapy. ${ }^{[5]}$

- Liposomal rifabutin is active against M.avium infection. ${ }^{[5]}$

- Liposomes are used for gene therapy such as Allovectin- $7^{\mathrm{TM}}$ for treatment of metastatic

- melanomaand colorectal carcinoma. ${ }^{[5]}$

- Leuprolide acetate liposomes are widely used in the intravenous administration of peptide drugs. ${ }^{[8]}$

- Liposomal antioxidants are widely used for the treatment of oxidative stress such as quercetin, Astaxanthin, Resveratrol ${ }^{[10]}$

- Liposomal verteporfin is used for the treatment of ocular histoplasmosis and subfoveal Choroidal neovascularization[CNV].$^{[12]}$

- Silica coated liposomes showed enhanced insulin delivery and very active in reducing the Glucose levels. ${ }^{[13]}$

- Stealth liposomes are used in vaccines, diagnostic imaging, inflammatory diseases, Gene transfection and targeted drug delivery. ${ }^{[14]}$

- Transferosomes are widely used for the treatment of skin diseases by employing corticosteroids. ${ }^{[15]}$

Table.3: List of Marketed Liposomal Products ${ }^{[5]}$

\begin{tabular}{|l|l|l|}
\hline Product name & Drug & Manufacturer [country] \\
\hline Abelcet & Amphotericin B & $\begin{array}{l}\text { The Liposome Company } \\
{[\mathrm{USA}]}\end{array}$ \\
\hline Allovectin-711 & HLA-B7 Plasmid & Vical Incorporation [USA] \\
\hline AmBisome & Amphotericin B & $\begin{array}{l}\text { NeXatar Pharmaceuticals } \\
{[\mathrm{USA}]}\end{array}$ \\
\hline Amphocil & Amphotericin B & $\begin{array}{l}\text { SEQUUS Pharmaceuticals } \\
{[\mathrm{USA}]}\end{array}$ \\
\hline DoxilT & Doxorubicin & $\begin{array}{l}\text { SEQUUS Pharmaceuticals } \\
\text { [USA] }\end{array}$ \\
\hline Doxosome & Doxorubicin & $\begin{array}{l}\text { Indian Institute of Chemical } \\
\text { Biology [India] }\end{array}$ \\
\hline L-AMP-LRC-1 & $\begin{array}{l}\text { Seth G.S. Medical College and } \\
\text { K.E.M. Hospital [India] }\end{array}$ \\
\hline MiKasomeTM & $\begin{array}{l}\text { NeXatar Pharmaceuticals } \\
\text { [USA] }\end{array}$ \\
\hline
\end{tabular}




\section{A Few Examples Of Past research investigations on liposomes}

- Eric A.Foressen et al., [1981] reported the study on the usage of anionic liposomes for the reduction of chronic doxorubicin induced cariotoxicity. ${ }^{[18]}$

- M.Schaller et al., [1996] reported the study on the interaction of liposomes with human Skin: the role of the stratum corneum. ${ }^{[19]}$

- Ahmed.H.Hikal et al.,[1997] reported the study on the preparation and evaluation of Acetazolamide liposomes as an ocular delivery system. ${ }^{[20]}$

- Chang-koo shim et al., [1997] reported the study on the invitro skin permeation of nicotine from proliposomes. $^{[21]}$

- Naoto Oku et al., [1999] reported the study on the anticancer therapy using glucuronate modified long circulating liposomes. ${ }^{[22]}$

- M. Brisaert et al., [2001] reported the study on liposomes with tretinoin: a physical and chemical evaluation. ${ }^{[23]}$

- Brian W. Barry et al., [2002] reported the study on Iontophoretic estradiol skin delivery and tritium exchange in ultra-deformable liposomes. ${ }^{[24]}$

- Luigi cattel et al., [2003] reported the study on preparation, characterization, cytotoxicity and pharmacokinetics of liposomes containing docetaxel. ${ }^{[25]}$

- AylaGursoy et al., [2004] reported the study on the co-encapsulation of isoniazid and rifampicin in liposomes and characterization of liposomes by derivative spectroscopy. ${ }^{[26]}$

- Dae-Duk Kim et al., [2006] reported the study on formulation of liposome for topical delivery of Arbutin. ${ }^{[27]}$

- YechezkelBarenholz et al.,[2009] reported the study on ultra sound triggered release of cisplatin from liposomes in murine tumours. ${ }^{[28]}$

\section{CONSTRAINTS OF LIPOSOMES}

The first constraint is the lipids, required for the production of liposomes are very scarce, should be of highly pure and expensive. The second one is instability of liposomes which requirespecialized storage conditions even if the products are freeze dried. ${ }^{[3]}$ The large scale production of liposomes is highly difficult as it involves the usage of large amounts of organic solvents in high concentrations which are not recommended as per regulatory norms. Even if it crosses all the barriers which are mentioned above, still the liposomal preparations encounter problems in the systemic circulation because they are recognized as foreign particlesand they will be up taken by the reticuloendothelial system. ${ }^{[2]}$

\section{CONCLUSION}

Liposomes are one of the unique drug delivery system, which can be of potential use in controlling and targeting drug delivery. These are administrated orally, parenterally and topically as well as used in cosmetic and hair technologies, sustained release formulations, diagnostic purpose and as good carriers in gene delivery .Various drugs with liposomaldelivery systems have been approved. Nowadays liposomes are used as versatile carriers for targeted delivery of drug.In a glance, this article reviewed the liposomes in brief starting from the historical evolution,types and methods of preparation, characterization, applicationsupto the availability of some marketed liposomal productsand various constraints regarding the formulation of liposomes. With the recent development in the field, several companiesare already actively engaged in expansion and evaluation of liposome products foranticancer, antifungal therapy and for prophylaxis. The future of drug therapeutics maynot lie in the development of new chemical entities but in the modification of the existingdrug molecules using liposomes to eliminatetoxicity and improve activity of the old drugs. Further refinements in the liposomal technology will spur the full-fledged evolvement of liposomes as drug carriers. 


\section{REFERENCES}

[1]. HimanshuAnwekar, Sitasharan Patel and A.K Singhai,Liposome - as drug carriers,IJPLS, Vol-2, Pg.No: $945-951$

[2]. Sharma Vijay K, Mishra D N, Sharma A K, Srivastava B, Liposomes: Present Prospective and Future Challenges, IJCPR, Vol-1, Pg.No: 1-16

[3]. MU Uhumwangho and RS Okor, Current trends in the production and biomedical applications of liposomes: A review,JMBR, Vol-4, Pg.No:9-21

[4]. Mohammad Riaz, Liposomes Preparation Methods,PJPS, Vol-19(1), Pg.No:65-77

[5]. ParveenGoyal,KumudGoyal,SengodanGurusamyVijaya Kumar, Ajit Singh, Om PrakashKatare,DinaNath Mishra, Liposomal drug delivery systems - Clinica Applications,Acta Pharm, Pg.No: 1-25.

[6]. C.Forbes, J.Wilby, G.Richardson, M.Sculpher,L.Mather,R.Riemsma, A Systematic Review and Economic evaluation of pegylated liposomal doxorubicin hydrochloride for ovarian cancer, HTA, Vol-6, No:23

[7]. AV Yadav, AS Shete, YN Gavane, AP Dabke, PV Kulkarni and SS Sakhare,Chitosomes: A Novel Drug Delivery System,RJPT, Vol-3, Pg.No: 4-12N. Arulsudar, N. Subramanian, Mishra.K, Chuttani,R.K. Sharma,and R.S.R. Murthy, Preparation, Characterization and Bio-distribution Study of Technetium-99m -Labeled Leuprolide Acetate-Loaded Liposomes in Ehrlich Ascites Tumor-Bearing Mice,AAPS PharmSci, 6 (1), Pg.No: 1-12

[8]. Suggy S. Chrai,R.Murari, Imran Ahmad, Liposomes : A Review Pharm Tech, Pg.No:28-34.

[9]. Zacharias E. Suntres, Liposomal Antioxidants for Protection against Oxidant-Induced Damage, Journal of Toxicology, Pg.No: 1-16

[10]. Upadhyay N, Mandal S, Bhatia L, Shailesh S, Chauhan P,A Review on "Ethosomes: An Emerging Approach for Drug Delivery through the Skin" Res Sci Tech, Pg.No:19-24

[11]. GyanP.Mishra, MahuyaBagui, Viral Tamboli, and AshimK.Mitra,Recent Applications of Liposomes in Ophthalmic Drug Delivery, Journal of Drug Delivery, Pg.No: 1-14

[12]. NeelamDwivedi, M. A. Arunagirinathan, Somesh Sharma, and JayeshBellare,Silica-Coated Liposomes for Insulin Delivery, Journal of Nanomaterials, Pg.No: 1-8

[13]. Katariasahil, sandhuPremjeet, Bilandi Ajay, AkankshaMiddha, KapoorBhawna,Stealth Liposomes: A Review, IJRAP, Pg.No: 1534-1538

[14]. P. R. Kulkarni, J. D. Yadav, K. A. Vaidya and P. P. Gandhi, Transferosomes: An Emerging Tool For Transdermal Drug Delivery,IJPSR, Vol-2(4), Pg.No:735-741

[15]. MalutaS.Mufamadi, VinessPillay, Yahya E. Choonara, Lisa C. DuToit,GirishModi, Dinesh Naidoo, and ValenceM. K. Ndesendo,A Review on Composite Liposomal Technologies for Specialized Drug Delivery, Journal of Drug Delivery, Pg.No: 1-19.

[16]. S.P.Vyas, R.K.KharTargeted \& Controlled Drug Delivery (VallabhPrakashan, Delhi, India,2007)

[17]. Eric.A.Forssen, ZoltanA.tokes, Proc.Natl. Usage of anionic liposomes for the reduction of chronic doxorubicin induced cariotoxicity, Acad.Sci.USA, Vol-78, Pg.No: 1873-1877

[18]. M.Schaller, H.C.Korting, Interaction of liposomes with human Skin: the role of the stratumCorneum, $A D D R$, Pg.No: 303-309

[19]. Ahmed.H.Hikal, Omaima.N.El-Gazayerly, Preparation and evaluation of Acetazolamide liposomes as an ocular delivery system,IJP, Pg.No: 121-127

[20]. Chang-koo Shim, Bo-Young Hwang,Byung-Hwajung, Suk-Jac Chung, Min-Hwa Lee, Invitro skin permeation of nicotine from proliposomes. JCR, Pg.No: 177-184.

[21]. Naoto Oku, Anticancer therapy using glucuronate modified long circulating liposomes, ADDR,Pg. No: 63-73

[22]. M.Brisaert, M.gabriels, V.Matthijs, J.Plaizier- Vercammen, Liposomes with tretinoin: A physical and chemical evaluation,JPBA, Pg.No: $909-917$

[23]. Brian.W.Barry, Ebetessam.A.Essa, Michael C.Bonner,Iontophoretic estradiol skin delivery and tritium exchange in ultra-deformable liposomes, IJP, Pg.No: 55-66

[24]. Luigi cattel, Maria Laura Immordino, Paola Brusa, Silvia Arpicco, Barbara Stella,FrancoDosio, Preparation, characterization, cytotoxicity and pharmacokinetics of liposomes containing docetaxel,JCR, Pg.No: 417-429

[25]. AylaGursoy, Ecekut, SumruOzkirimli, Co-encapsulation of isoniazid and ifampicin in liposomes and characterization of liposomes by derivative spectroscopy, IJP, Pg.No: 115-123

[26]. Dae-Duk Kim, Ai-Hua Wen, Min-Koo Choi, Formulation of liposome for topical delivery of Arbutin,Arch Pharm Res, Vol-29, Pg.No: 1187-1192

[27]. YechezkelBarenholz, AviScroeder, Reuma Honen, KerenTurjeman,Alberto Gabizon,JosephKost, Ultra sound triggered release of cisplatin from liposomes in murinetumours, JCR, Pg.No: 63-68 